\title{
Editorial
}

\section{Klatskin Tumors: Challenging as Ever}

\author{
Karl J. Oldhafer ${ }^{a, b}$ Thomas von Hahn ${ }^{b, c}$ \\ aDivision of Hepatobiliary and Pancreatic Surgery, Department of Surgery, Asklepios Hospital Barmbek,

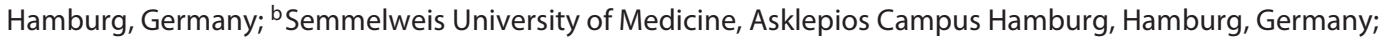 \\ 'Department of Gastroenterology, Hepatology, and Interventional Endoscopy, Asklepios Hospital Barmbek, \\ Hamburg, Germany
}

\section{Keywords \\ Perihilar cholangiocarcinoma · Hepatectomy · Endoscopic retrograde cholangiography - Cholangioscopy}

Gerald Klatskin described in 1965 in the American Journal of Medicine the distinctive and pathological features of thirteen cases with adenocarcinoma of the hepatic duct at its bifurcation within the porta hepatis [1]. Due to several unique features these so-called Klatskin tumors (or perihilar cholangiocarcinomas) remain among the most challenging neoplasms of the gastrointestinal tract for radiologists, gastroenterologists, and liver surgeons. Clinical outcomes have improved somewhat since Klatskin first defined the entity, but there is still need for better diagnostic and therapeutic options. In this special issue of Visceral Medicine we address the key contemporary challenges.

More than 50 years after Klatskin's first description, a lot of new information about this type of tumor has become available. For instance, several risk factors have been identified, such as primary sclerosing cholangitis, liver fluke infection, type 2 diabetes, obesity, hepatitis B, hepatitis C, and cirrhosis. However, Klatskin's thoughts are still noteworthy. He concluded that:

I. Tumors of this type are frequently overlooked during laparotomy because of failure to explore the hepatic duct bifurcation and its tributaries by retrograde probing and cholangiography.

Even with modern imaging technologies available today, perihilar tumors still remain a diagnostic challenge. Solonitsyn and Dechêne [2] argue in this issue that in sus- pected perihilar malignancy a mosaic of clinical data has to be taken into account. There is no single imaging method offering sufficient diagnostic accuracy. Magnetic resonance cholangiography (MRC) allows delineation of the complete biliary anatomy. Ideally, MRC should be performed before stenting of the biliary system. Endoscopic retrograde cholangiography (ERC) is superior to MRC in terms of resolution, but complete visualization of the biliary tree is difficult to achieve. The use of cholangioscopy with modular small-caliber scopes to visualize the tumor directly seems promising. However, it is well known that bile duct cancer can spread beneath the surface along periductal structures or produce seemingly discontinuous "skip" lesions. Therefore, the accuracy of this method is limited. Reliable biomarkers are still lacking. Brush cytology, EUS-guided fine needle aspiration, and intraductal biopsy all have limited sensitivity of around $50 \%$ and thus cannot rule out malignancy. Bile samples could be an interesting source for early detection and diagnosis in the future; both proteomic and microRNA-based assays are under evaluation.

II. Death in this disease is usually attributable to hepatocellular failure and/or hepatobiliary infection secondary to unrelieved biliary obstruction rather than to massive invasion of the liver by tumor or to extrahepatic metastases.

This remains true especially after successful radical liver resection. Evaluation of liver function before hepatectomy is key for an uneventful postoperative course. In this issue, Yuzo Yamamoto [3] reviews the currently available liver function tests. Clinical, standard laboratory, and ul- 
trasound assessment of liver morphology and function remain a cornerstone, but more advanced methods may provide much needed additional predictors to assess feasibility prior to major resections. These include elastography, hepatovenous portal pressure gradient measurement, indocyanine green clearance, or ${ }^{13} \mathrm{C}$-methacetin metabolism-based assays.

III. Palliative surgery aimed at relieving biliary obstruction may restore the patient to a good state of health for a remarkably long period of time.

In the 1960s surgery was only performed for palliation. Later, first bile duct resections together with partial liver resections were undertaken, but morbidity and mortality were very high. Up to now, liver resections for perihilar tumors are the most challenging among liver tumors. However, surgical therapy offers the only chance for cure. Lyann Nooijen et al. [4] describe in this special issue surgical strategies for perihilar cholangiocarcinoma, including the role of preoperative induction of hypertrophy. Despite these advances in surgical technique there is still a high rate of recurrence. This underlines the need for adjuvant therapy concepts. In this issue Arndt Vogel and Anna Saborowski [5] describe the positive effect of present adjuvant chemotherapy and give an overview of systemic therapy options for other disease phases as well. In some cases patients suffering from recurrent cholangiocarcinoma after resection may be candidates for repeated surgery. Hauke Lang [6] describes that in selected patients radical resection is possible and recommends that repeated surgical treatment be attempted whenever possible.
$I V$. Such palliation may be achieved by internal drainage of only one of the major intrahepatic bile ducts.

For palliation adequate biliary drainage is very important in order to improve the patient's quality of life and to reduce the risk of obstructive cholangitis. ERC with placement of plastic or self-expanding metal stents remains the standard approach. Where ERC is not possible a emerging option is endoscopic ultrasound-guided internal biliary drainage. Moreover, local ablative therapies are available to improve local tumor control and to secure biliary drainage. Tobias Weismüller [7] describes the new method of intraductal RFA in this special issue.

Finally, the guest editors would like to express their sincere gratitude to the authors involved who provided such valuable contributions to this special topic on perihilar cholangiocarcinoma and the journal's editorial team for their support.

\section{Conflict of Interest Statement}

The authors have no conflicts of interest to declare.

\section{Funding Sources}

None.

\section{Author Contributions}

K.J. Oldhafer and T. von Hahn jointly planned, wrote, and revised this editorial.

\section{References}

1 Klatskin G. Adenocarcinoma of the hepatic duct at its bifurcation within the porta hepatis. An unusual tumor with distinctive clinical and pathological features. Am J Med. 1965 Feb;38:241-56.

2 Solonitsyn E, Dechêne A. Diagnostic approach to suspected perihilar malignancy. Visc Med. 2020 doi: 10.1159/000513950 [Epub ahead of print].
3 Yamamoto Y. Evaluation of liver function and the role of biliary drainage before major hepatic resections. Visc Med. 2020 doi: 10.1159/000512439 [Epub ahead of print].

4 Nooijen LE, Swijnenburg RJ, Klümpen HJ, Verheij J, Kazemier G, van Gulik TM, et al. Surgical therapy for perihilar cholangiocarcinoma: state of the art. Visc Med. 2020 doi: 10.1159/000514032 [Epub ahead of print].
5 Vogel A, Saborowski A. Current and future systemic therapies in biliary tract cancer. Visc Med. 2020 doi: 10.1159/000513969 [Epub ahead of print].

6 Lang H. Surgical approach to recurrent cholangiocarcinoma. Visc Med. 2020 doi: 10.1159/ 000513711 [Epub ahead of print].

7 Weismüller TJ. Role of intraductal RFA: a novel tool in the palliative care of perihilar cholangiocarcinoma. Visc Med. 2020 doi: 10.1159/000513970 [Epub ahead of print]. 